\title{
Resolving the inner disk of UX Orionis ${ }^{\star}$
}

\author{
A. Kreplin ${ }^{1,2}$, D. Madlener ${ }^{2}$, L. Chen ${ }^{2}$, G. Weigelt ${ }^{2}$, S. Kraus ${ }^{1}$, V. Grinin ${ }^{3,4}$, L. Tambovtseva ${ }^{3}$, and and M. Kishimoto ${ }^{5}$ \\ ${ }^{1}$ University of Exeter, Astrophysics Group, Stocker Road, Exeter, EX4 4QL, UK \\ e-mail: akreplin@astro.ex.ac.uk \\ 2 Max-Planck-Institut für Radioastronomie, Auf dem Hügel 69, 53121 Bonn, Germany \\ 3 Pulkovo Observatory of RAS, Pulkovskoe shosse 65, 196140 St. Petersburg, Russia \\ 4 V.V. Sobolev Astronomical Institute, St. Petersburg University, 196140 St. Petersburg, Russia \\ 5 Kyoto Sangyo University, Motoyama, Kamigamo, 603-8555 Kita-ku, Kyoto, Japan
}

Received 9 February 2016 / Accepted 5 April 2016

\begin{abstract}
Aims. The cause of the UX Ori variability in some Herbig Ae/Be stars is still a matter of debate. Detailed studies of the circumstellar environment of UX Ori objects (UXORs) are required to test the hypothesis that the observed drop in photometry might be related to obscuration events.

Methods. Using near- and mid-infrared interferometric AMBER and MIDI observations, we resolved the inner circumstellar disk region around UX Ori.

Results. We fitted the $\mathrm{K}$-, $\mathrm{H}$-, and $\mathrm{N}$-band visibilities and the spectral energy distribution (SED) of UX Ori with geometric and parametric disk models. The best-fit $K$-band geometric model consists of an inclined ring and a halo component. We obtained a ring-fit radius of $0.45 \pm 0.07 \mathrm{AU}$ (at a distance of $460 \mathrm{pc}$ ), an inclination of $55.6 \pm 2.4^{\circ}$, a position angle of the system axis of $127.5 \pm 24.5^{\circ}$, and a flux contribution of the over-resolved halo component to the total near-infrared excess of $16.8 \pm 4.1 \%$. The best-fit $N$-band model consists of an elongated Gaussian with a $H W H M \sim 5 \mathrm{AU}$ of the semi-major axis and an axis ration of $a / b \sim 3.4$ (corresponding to an inclination of $\sim 72^{\circ}$ ). With a parametric disk model, we fitted all near- and mid-infrared visibilities and the SED simultaneously. The model disk starts at an inner radius of $0.46 \pm 0.06 \mathrm{AU}$ with an inner rim temperature of $1498 \pm 70 \mathrm{~K}$. The disk is seen under an nearly edge-on inclination of $70 \pm 5^{\circ}$. This supports any theories that require high-inclination angles to explain obscuration events in the line of sight to the observer, for example, in UX Ori objects where orbiting dust clouds in the disk or disk atmosphere can obscure the central star.
\end{abstract}

Key words. techniques: interferometric - stars: pre-main sequence - stars: variables: T Tauri, Herbig $\mathrm{Ae} / \mathrm{Be}-$ stars: individual: UX Ori

\section{Introduction}

The UX Ori type stars (or UXORs) are a class of photometrically active young objects, predominantly Herbig Ae stars and some amount of young F and G stars (see, e.g., Herbst et al. 1994). The typical light curve of UXORs exhibits aperiodic algol-like minima with a typical $V$-band drop of $\Delta V \sim 2-3$ mag with a duration from a few to a few tens of days. The brightness minima are accompanied with an increase of the linear polarizations and spectroscopic variations (see review by Grinin 2000, and references therein). Several different explanations for this variability have been suggested. One explanation assumes that the brightness variations are linked to obscurations of the central star by orbiting dust clouds in an almost edge-on disk (Grinin et al. 1991; Natta et al. 1997). Dynamical effects like gravitating bodies might also lead to regular perturbations of the density structure in the disk (see, e.g., de Wit et al. 2013). Turbulent filaments at the location of the puffed-up inner rim might create obscuration events (Dullemond et al. 2003). The clumps of dust in the dusty outflow may occasionally intercept the line of sight (Vinković \& Jurkić 2007). The model of the variable circumstellar (CS) extinction with the account of scattered radiation (Grinin 1988) gives the most natural explanation of the observed

\footnotetext{
* Based on observations made with ESO telescopes at Paranal Observatory under program IDs: 090.C-0769, 074.C-0552.
}

phenomena. The high linear polarization observed systematically during the deep minima motivated us to assume that the violent photometric activity of UXORs is a result of the low inclination of the CS disks to the line of sight (Grinin et al. 1991). A different explanation of the UXOR physics was suggested by Herbst \& Shevchenko (1999). They suggested that the photometric activity of UXORs does not depend on the disk orientation and is a result of the FUOR-like activity caused by the strong and unstable accretion activity of these stars.

Fortunately, interferometric observations of young stars permit us to measure the inclination of their CS disks relative to the line of sight. Detailed studies on the geometry and shape of the circumstellar dust distribution around UXORs are important to improve our understanding of the origin of the strong photometric variability. Therefore, we conducted infrared interferometric observations of UX Orionis, the prototype star of this sub-class of variable Herbig Ae/Be stars that allow us to resolve the structure of the inner disk on milliarcsecond (mas) scale.

UX Ori is a well-studied Herbig Ae star with spectral type A3, located at a distance of $\sim 460 \mathrm{pc}$, and it has a stellar mass of 2.3-2.5 $M_{\odot}$ (Rostopchina 1999; Natta et al. 1999). Its stellar age estimates range from 2 to $4.8 \mathrm{Myr}$ (Natta et al. 1999; Testi et al. 2001; Manoj et al. 2006). Millimeter interferometry suggests the existence of a compact circumstellar disk (<100 AU; Natta et al. 1999). Polarimetric observations 
Table 1. Observation log of the VLTI/AMBER and VLTI/MIDI observations of UX Ori.

\begin{tabular}{|c|c|c|c|c|c|c|c|c|c|}
\hline Intrument & Date & Telescopes & $\begin{array}{c}\mathrm{UT} \\
{[\mathrm{hh}: \mathrm{mm}]}\end{array}$ & $\begin{array}{l}\text { DIT } \\
{[\mathrm{ms}]}\end{array}$ & $\begin{array}{c}\text { NDIT } \\
\#\end{array}$ & $\begin{array}{l}\text { Proj. baselines } \\
{[\mathrm{m}]}\end{array}$ & $\begin{array}{l}\mathrm{PA} \\
{\left[{ }^{\circ}\right]}\end{array}$ & Calibrator & $\begin{array}{l}\text { Diam. } \\
\text { [mas] }\end{array}$ \\
\hline \multirow[t]{5}{*}{ AMBER } & $2012-12-30$ & UT2/UT3/UT4 & $00: 45$ & 50 & 8000 & $38.0 / 56.5 / 71.5$ & $25.5 / 109.1 / 77.2$ & HD 30521 & $0.0 \pm 0.04^{a}$ \\
\hline & " & " & $01: 45$ & & 5000 & $41.0 / 61.1 / 82.1$ & $33.9 / 108.5 / 79.7$ & HD 34909 & $0.32 \pm 0.03^{a}$ \\
\hline & $\prime \prime$ & " & $02: 17$ & " & 6000 & 42.9 / 62.3 / 86.7 & $38.2 / 108.7 / 80.9$ & HD 33208 & $0.27 \pm 0.03^{a}$ \\
\hline & " & " & $03: 11$ & " & 5000 & $45.0 / 61.8 / 89.4$ & $42.3 / 109.7 / 82.0$ & HD 33208 & " \\
\hline & " & " & 03:45 & " & 6000 & $46.0 / 60.0 / 88.9$ & $44.2 / 110.9 / 82.5$ & HD 33208 & " \\
\hline \multirow[t]{5}{*}{ MIDI } & $2004-12-28$ & UT2/UT3 & $06: 25$ & - & - & 44.5 & 44.8 & HD 37160 & $2.20 \pm 0.02^{b}$ \\
\hline & $2004-12-30$ & UT3/UT4 & 07:02 & - & - & 32.9 & 136.0 & & \\
\hline & $2007-10-25$ & UT2/UT3 & $07: 34$ & - & - & 44.9 & 42.1 & HD 31767 & $2.73 \pm 0.04^{b}$ \\
\hline & " & UT2/UT3 & $07: 49$ & - & - & 45.5 & 43.1 & " & " \\
\hline & 2007-11-23 & UT1/UT4 & 03:09 & - & - & 97.7 & 48.6 & & " \\
\hline
\end{tabular}

Notes. ${ }^{(a)}$ The calibrator uniform disk (UD) diameter ( $K$ band) was taken from the JMMC Stellar Diameter Catalogue (Lafrasse et al. 2010). ${ }^{(b)}$ The calibrator limb-darkened disk (LDD) diameter was taken from the Borde Catalogue (Borde et al. 2002).

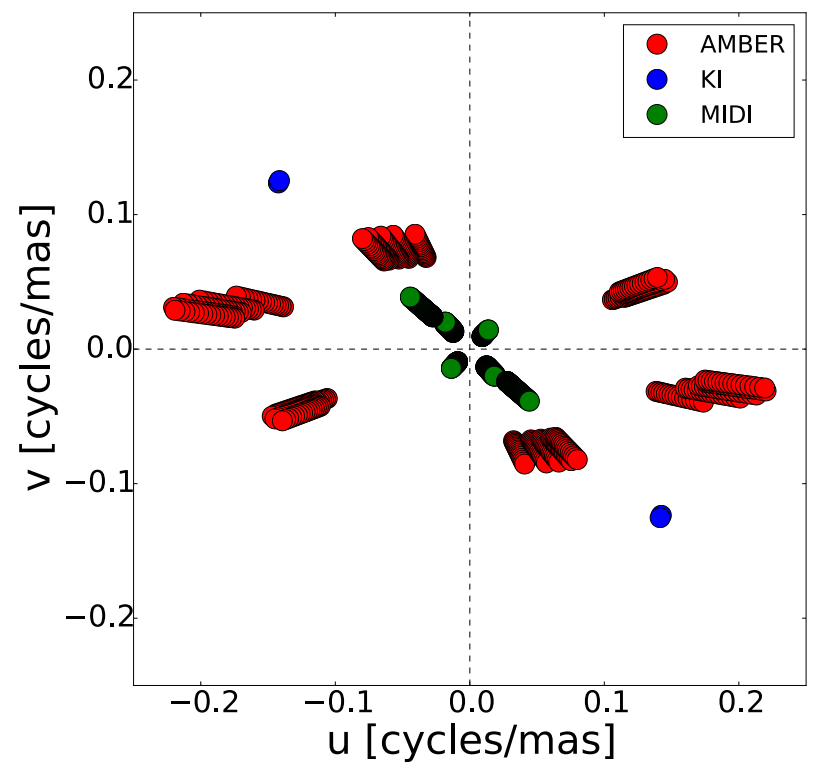

Fig. 1. $u v$ coverage of our AMBER measurements (red), the archival MIDI measurements (green), and the KI measurements from the literature (blue).

during deep minima showed a linear $V$-band polarization angle of $125.5-128.7^{\circ}$ (Voshchinnikov et al. 1988). The modeling of HIPPARCos data lead to evidence of a binary nature of UX Ori and predicted a binary separation of $\sim 20$ mas with a position angle (PA) of $\sim 260^{\circ}$ (Bertout et al. 1999), which remains undetected by the spectro-astrometric survey of binary candidates by Wheelwright et al. (2010).

In this paper, we present near- and mid-infrared interferometric observations of the Herbig Ae star UX Ori. In Sect. 2 we describe our observations and the data reduction. In Sect. 3 we discuss our geometric and passive flared-disk modeling and the derived disk inclination of UX Ori. We show the results in Sect. 4 and present a conclusion in Sect. 5.

\section{Observations and data reduction}

We observed UX Ori with the AMBER instrument of the Very Large Telescope Interferometer (VLTI) using the UT configuration UT2-UT3-UT4 in December 2012 (see $u v$ coverage in Fig. 1; red points). The AMBER instrument is a threebeam combiner that records spectrally dispersed three-beam interferograms (Petrov et al. 2007). We recorded $H$ - and $K$-band interferograms in the low spectral resolution mode $(R \sim 35)$ with a detector integration time (DIT) of $50 \mathrm{~ms}$. For data reduction, we employed the software library amdlib v3.0.5 ${ }^{1}$. We selected $20 \%$ of the frames with the highest fringe signal-to-noise ratio (S/N; Tatulli et al. 2007) and applied a histogram equalization method for piston correction (Kreplin et al. 2012). The observation $\log$ is shown in Table 1. For modeling and interpretation, we complemented our near-infrared AMBER data with archival mid-infrared MIDI data (published in the survey paper of Menu et al. 2015). We re-reduced this data set of five MIDI measurements using custom IDL routines (Kishimoto et al. 2011) that incorporates parts of the standard software EWS ${ }^{2}$. In addition, we included $K$-band measurements of UX Ori with the Keck Interferometer (KI) from the literature (Monnier et al. 2005), illustrated in the $u v$ coverage in Fig. 1 by green and blue points, respectively.

\section{Modeling}

To characterize the size and shape of the near- and mid-infrared emitting region of UX Ori, we fit geometric ring and Gaussian models to the observed visibilities. Because the measured AMBER closure phases are equal to zero (see Fig. 2), consistent with a centro-symmetric brightness distributions, we did not use them in further modeling steps since we exclusively applied centrosymmetric models in this paper.

The visibilities depend on both the flux of the unresolved central star and the circumstellar structure. Therefore, we first derived the visibility $V_{\mathrm{CS}}$ of the circumstellar environment only (i.e., exclusive of the central star)

$V_{\mathrm{CS}}=\frac{\left|V_{\text {meas }}\left(F_{\star}+F_{\mathrm{CS}}\right)-F_{\star} V_{\star}\right|}{F_{\mathrm{CS}}}$,

where $F_{\star}$ is the stellar flux, $F_{\mathrm{CS}}$ the circumstellar flux component, $V_{\star}$ the stellar visibility, and $V_{\text {meas }}$ the measured visibility. We set $V_{\star}=1$ because the central star is unresolved by the baselines used in our observation (stellar radius $\sim 0.03$ mas at $d=460$ pc; Rostopchina 1999). The circumstellar-to-stellar flux ratio was derived for every spectral channel by fitting a Kurucz model (Kurucz 1979) to the spectral energy distribution (SED)

\footnotetext{
1 The AMBER reduction package amdlib is available at: http:// wWw. jmmc.fr/data_processing_amber.htm

2 The MIDI reduction package $M I A+E W S$ is available at: http://
} home. strw . leidenuniv.nl/ nevec/MIDI/index . html 


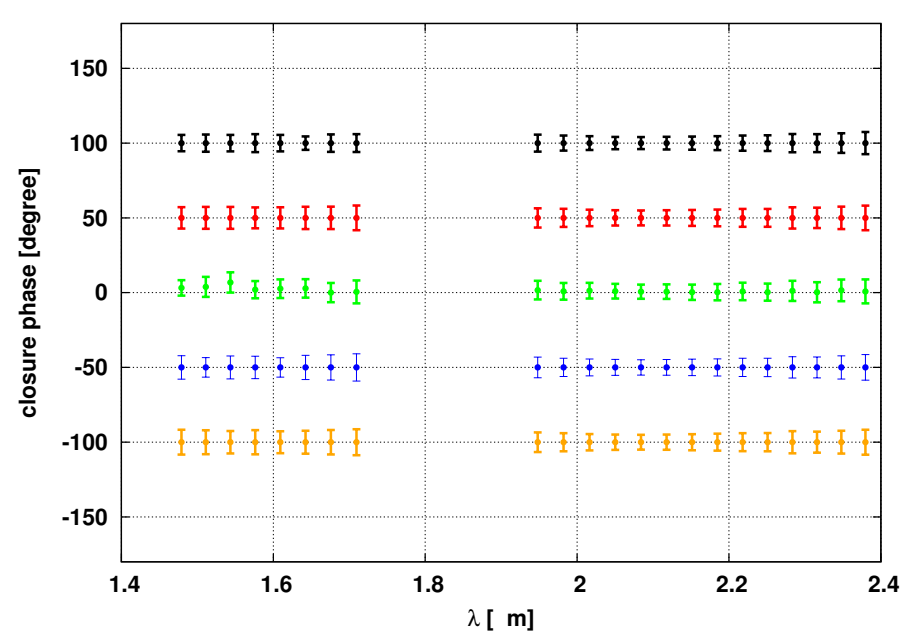

Fig. 2. Closure phases of the five AMBER measurements of UX Ori (see Table 1). For clarity, we separated the different data sets by 50 degree. All closure phases are close to zero, consistent with a centro-symmetric brightness distribution.

data (Natta et al. 1999) of UX Ori. Because of the variable nature of the $V$-band flux of UX Ori, we took simultaneous photometric observations from 2012 December 18-30 to determine its brightness state. With a $V$ magnitude between 10.5 and 9.5, UX Ori was found to be in a bright state during our near-infrared interferometric observations. For the fitting we used a Kurucz model with the following stellar parameters: $T_{\text {eff }}=8600 \mathrm{~K}, \log (g)=4$, $\log (Z / H)=-2.5$ for a stellar radius of $R_{\star}=2.7 R_{\odot}$ at a distance of $d=460 \mathrm{pc}$ (Rostopchina 1999). For the central wavelength in our $H$ - and $K$-band data, we derived $F_{\mathrm{CS}} / F_{\star}=5.5$ for $\lambda=2.2 \mu \mathrm{m}$ and $F_{\mathrm{CS}} / F_{\star}=2.8$ for $\lambda=1.7 \mu \mathrm{m}$. In Fig. 3 we plot the visibilities $V_{\mathrm{CS}}$.

\subsection{Geometric models}

\subsubsection{AMBER data}

We first fit a simple geometric ring model with a fractional ring width of $20 \%$ of the inner ring radius and with an inclination angle of $0^{\circ}$ (i.e., seen face-on) to the near-infrared AMBER data. However, no successful fit to the $K$ - and $H$-band data could be obtained (see high $\chi^{2}$ values in Table 2). Therefore, we decided to increase the complexity of the model and the number of free parameters (labeled \#p in Table 2) step by step to find a bestfit model with the lowest number of parameters. We first added a completely over-resolved halo component (i.e., $V_{\text {halo }}=0$ ) to the above ring model. The flux ratio $f_{\mathrm{h}}=F_{\text {halo }} /\left(F_{\mathrm{CS}}+F_{\text {halo }}\right)$ between the flux $F_{\text {halo }}$ of the extended halo component and the total model flux leads to only one additional free parameter. In further steps, the size of the halo component is also a free parameter. To account for this extended component, we described the model visibility $V_{\text {model }}$ by

$V_{\text {model }}=\frac{\left|F_{\mathrm{CS}} V_{\mathrm{CS}}+F_{\text {halo }} V_{\text {halo }}\right|}{F_{\mathrm{CS}}+F_{\text {halo }}}$,

where $F_{\text {halo }}$ and $V_{\text {halo }}$ denote the flux and visibility of the halo component, respectively. We modeled different types of halos (see Table 2). First, we assumed the halo to be circularsymmetric and later to have the same elongation (due to inclination effects) and PA as the inclined ring component (called elongated halo in Table 2). In Table 2, the number of free parameters increases from the top to bottom (for each $\mathrm{H}$ and $K$-individually; see $\# \mathrm{p}$ in Col. 8). By introducing an over-resolved halo component, the $\chi_{\text {red }}^{2}$ value could be significantly reduced in $K$ from 8.14 to 1.63 and in $H$ from 22.93 to 8.05. The application of an inclined ring, with the three free parameters, radius $r$, inclination $\theta_{\text {geo,NIR }}$, and the position angle of the semi-minor axis $\Phi_{\text {geo,NIR }}$, led to a similar good fit. Only the combination of an inclined ring and an over-resolved halo component provides $\chi_{\text {red }}^{2}$ values close to 1 (see last three $K$-band models in Table 2). The $H$-band fit does not achieve such low $\chi^{2}$ values since the fit is more sensitive to the errors of the assumed stellar model and the $H$-band data suffer in general from a lower SNR. In summary, we found that a single ring, a single inclined ring or a ring + halo model were unable to fit the near-infrared visibilities well. By introducing an over-resolved component to the inclined ring model, the corresponding $\chi^{2}$ values are significantly reduced (see last three models in Table 2). The best fits are obtained with an inclined ring plus one of the halo models. The inclined ring plus elongated halo model is marginally the best and further work is based on that. The best-fit parameters of these two-dimensional geometric models are summarized in Table 2.

\subsubsection{MIDI data}

To compare the near-infrared emitting structure of the hot inner disk regions of UX Ori to the outer mid-infrared disk structure, we first estimated the characteristic size of the mid-infraredemitting region by fitting a two-dimensional Gaussian distribution. The free parameters are the size (half width at half maximum, HWHM), the elongation ratio $(a / b)$ of the semi-major and -minor axis, and the position angle of the semi-minor axis

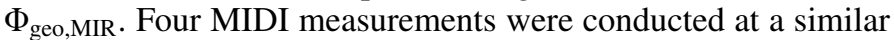
PA between $\sim 42-49^{\circ}$ and one measurement at an approximately orthogonal oriented configuration at $136^{\circ}$ (see green points in Fig. 1). With data at only two independent PAs in the $u v$-plane, we were unable to simultaneously fit the PA, the HWHM, and the elongation ratio $a / b$. Therefore, we assumed that the midinfrared emission arises from the same physical structure as in the near-infrared and fixed the model PA $\Phi_{\text {geo,MIR }}=127.5^{\circ}$ to the near-infrared value. From the two-dimensional Gaussian fit to the MIDI visibilities, we derived a $H W H M=5.1 \pm 0.9 \mathrm{AU}$. The derived size ratio of $a / b=3.4 \pm 1.1$ suggests an elongated structure, possibly an inclined disk structure (corresponding to an inclination of $72.9 \pm 17.9^{\circ}$ ).

\subsection{Disk model}

We employed a simple modified Chiang \& Goldreich model (Chiang \& Goldreich 1997) to compute the visibilities and the SED of a flared irradiated passive circumstellar dust+gas disk with an inner hole (hereafter DDN model; Dullemond et al. 2001). Our model makes use of the cgplus software ${ }^{3}$ developed by Dullemond and includes the modifications described in Dullemond et al. (2001) and Dominik et al. (2003). We fit the SED and visibilities simultaneously. The disk starts at an inner radius where the disk has the dust sublimation temperature $T_{\text {in }}$. The model visibilities were computed by projecting the intensity distribution of the DDN model to inclined rings corresponding to the inclination angle. For the central star, we used the same stellar parameters as described in Sect. 3 and kept them

\footnotetext{
3 The cgplus software is available at: http://www.mpia.de/
} $\sim$ dullemon/radtrans/fitcgplus/index.html 


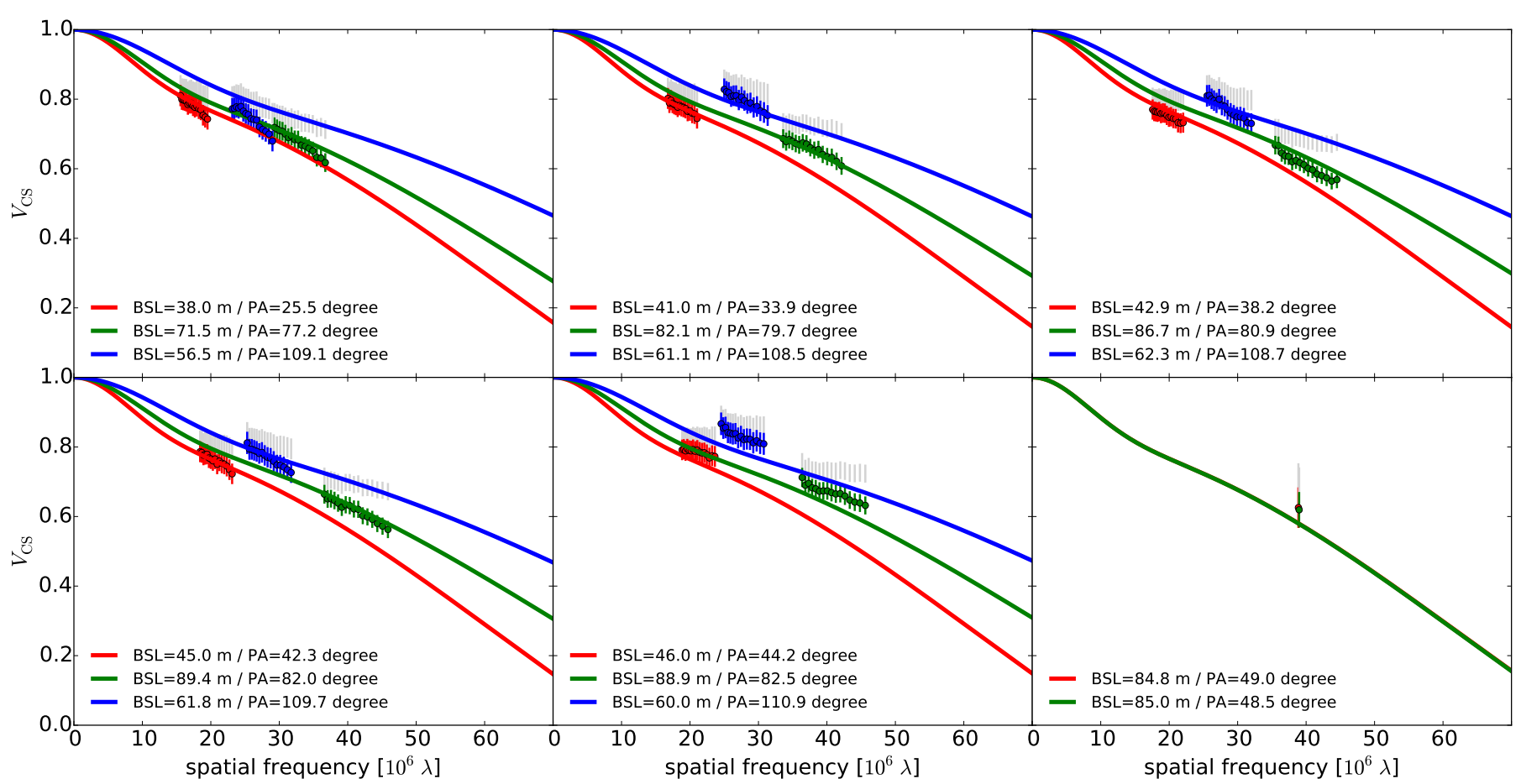

Fig. 3. Two-dimensional fit (colored lines) of a geometric model consisting of an inclined ring and a halo (see Table 2: inclined ring + elongated halo). The fitting included all derived $K$-band visibilities $V_{\mathrm{CS}}$ (colored data points) of the circumstellar environment (i.e., exclusive of the central star; Eq. (1)), in other words, we fitted this two-dimensional ring + elongated halo model to all visibilities instead of fitting one-dimensional visibility models to the visibilities corresponding to a single PA. In gray we plot the visibility data $V_{\text {meas. }}$. All except the bottom right panel show the AMBER observations. The bottom right panel shows the KI measurement from Monnier et al. (2005). The two KI measurements are close together in position angle and baseline length and lie on top of each other and of the model curves.

Table 2. Best-fit geometric models of the $K$ - and $H$-band visibilities.

\begin{tabular}{|c|c|c|c|c|c|c|c|c|}
\hline Band & Type & $\begin{array}{c}\text { Radius } r \\
\text { [AU] }\end{array}$ & $\begin{array}{c}\theta_{\text {geo,NIR }}{ }^{1} \\
{\left[^{\circ}\right]}\end{array}$ & $\begin{array}{c}\Phi_{\text {geo,NIR }}{ }^{2} \\
{\left[^{\circ}\right]}\end{array}$ & $\begin{array}{c}f_{\mathrm{h}} \\
{[\%]}\end{array}$ & $\begin{array}{c}H W H M^{3} \\
{[\mathrm{AU}]}\end{array}$ & $\# p^{4}$ & $\chi_{\text {red }}^{2}$ \\
\hline$K$ & ring & $0.51 \pm 0.01$ & - & - & - & - & 1 & 8.14 \\
\hline " & ring + halo & $.38 \pm 0.01$ & - & - & $15.8 \pm 2.4$ & $\infty$ & 2 & 1.63 \\
\hline$\prime \prime$ & inclined ring & $0.88 \pm 0.02$ & $70.1 \pm 0.6$ & $91.4 \pm 1.5$ & - & - & 3 & 1.63 \\
\hline in & nclined ring + halo (infinite size) & $0.46 \pm 0.19$ & $55.6 \pm 2.0$ & $131.8 \pm 27.3$ & $15.9 \pm 3.3$ & $\infty$ & 4 & 0.82 \\
\hline$\prime \prime$ & inclined ring + halo (finite size) & $0.46 \pm 0.25$ & $55.6 \pm 2.1$ & $131.8 \pm 27.3$ & $16.5 \pm 3.4$ & $>7.6$ & 5 & 0.83 \\
\hline "I & inclined ring + elongated halo & $0.45 \pm 0.07$ & $55.6 \pm 2.4$ & $127.5 \pm 24.5$ & $16.8 \pm 4.1$ & $>4.8$ & 5 & 0.81 \\
\hline$H$ & ring & $0.48 \pm 0.01$ & - & - & - & - & 1 & 22.93 \\
\hline$"$ & ring + halo & $0.35 \pm 0.01$ & - & - & $26.8 \pm 0.01$ & $\infty$ & 2 & 8.05 \\
\hline$\prime \prime$ & inclined ring & $0.84 \pm 0.02$ & $70.2 \pm 0.8$ & $90.0 \pm 1.4$ & - & - & 3 & 8.09 \\
\hline in & nclined ring + halo (infinite size) & $0.38 \pm 0.10$ & $>55.0$ & $147.4 \pm 31.17$ & $29.6 \pm 7.2$ & $\infty$ & 4 & 6.99 \\
\hline$\prime \prime$ & inclined ring + halo (finite size) & $0.38 \pm 0.09$ & $>51.8$ & $147.5 \pm 31.2$ & $29.6 \pm 7.3$ & $>14.0$ & 5 & 7.05 \\
\hline$\prime \prime$ & inclined ring + elongated halo & $0.38 \pm 0.08$ & $48.7 \pm 2.3$ & $147.4 \pm 20.2$ & $29.6 \pm 7.3$ & $>18.6$ & 5 & 7.05 \\
\hline
\end{tabular}

Notes. ${ }^{(1)}$ Inclination angle. ${ }^{(2)}$ Position angle of the model semi-major axis. ${ }^{(3)}$ Semi-major axis $a .{ }^{(4)}$ Number of free fit-parameters.

fixed during the fitting process $\left(T_{\text {eff }}=8600 \mathrm{~K}, \log (g)=4\right.$, $\log (Z / H)=-2.5$, stellar radius of $R_{\star}=2.7 R_{\odot}$ at a distance of $d=460 \mathrm{pc})$. For simplicity, we used pure astronomical silicates (Draine \& Lee 1984) with a interstellar dust grain size distribution with $n(a) \sim a^{-3.5}$ (Mathis et al. 1977) with grains sizes from $a_{\min }=0.01 \mu \mathrm{m}$ to $a_{\max }=1.0 \mu \mathrm{m}$ (Reimann et al. 1997; Natta et al. 1999), required to fit the strong $10 \mu \mathrm{m}$ silicate feature seen in the MIDI spectrum of UX Ori (see bottom panel in Fig. 4). With this fixed chemistry and set of stellar parameters, the DDN model can be described by seven free parameters, the inner rim temperature $T_{\text {in }}$, the outer radius of the disk $R_{\text {out }}$, the scale height of the inner rim, controlled by the dimensionless parameter $\chi$, the mass of the disk (gas + dust) $M_{\text {disk }}$, the exponent of the surface density distribution $\beta$, the inclination angle $\theta_{\mathrm{DDN}}$, and PA of the disk system axes $\phi_{\mathrm{DDN}}$. The inner radius $R_{\text {in }}$ is not a free parameter but was computed from $T_{\text {in }}$ following Eq. (14) in Dullemond et al. (2001). To account for the required extended halo found in the geometric modeling, we introduced an overresolved component (with $V_{\text {halo }}=0$ ) to the model. We assume that the halo scatters the light from the star and the inner rim 
Table 3. Best-fit parameters of the DDN-model.

\begin{tabular}{cccccccc}
\hline \hline $\begin{array}{c}T_{\text {in }} \\
{[\mathrm{K}]}\end{array}$ & $\begin{array}{c}R_{\text {out }} \\
{[\mathrm{AU}]}\end{array}$ & $\beta$ & $\chi$ & $\begin{array}{c}M_{\text {disk }} \\
{\left[M_{\odot}\right]}\end{array}$ & $f_{\mathrm{h}}$ & $\begin{array}{c}\theta_{\mathrm{DDN}} \\
{\left[{ }^{\circ}\right]}\end{array}$ & $\begin{array}{c}\Phi_{\text {DDN }} \\
{\left[{ }^{\circ}\right]}\end{array}$ \\
\hline $1498 \pm 70$ & $25.9 \pm 5.8$ & $-1.79 \pm 0.12$ & $4.02 \pm 0.12$ & $0.60 \pm 0.04$ & $0.13 \pm 0.02$ & $70 \pm 5$ & $133 \pm 5$ \\
\hline
\end{tabular}

(see, e.g., Chen et al. 2012). This introduces one additional parameter, the halo flux ratio $f_{\mathrm{h}}$ that defines the emitted halo flux $F_{\text {halo }}=f_{\mathrm{h}}\left(F_{\star}+F_{\text {rim }}\right)$, where $F_{\star}$ denotes the stellar flux and $F_{\text {rim }}$ the flux emitted from the inner rim. Our final model therefore incorporates eight free parameters. The best-fit parameters are summarized in Table 3.

To calculate the corresponding error of each parameter, we used bootstrapping to create 600 new realizations of our data sets and applied a model fit with the same starting parameters (motivated by our geometric model results). Each fit corresponds to a new best-fit parameter set. The spread of the parameter values is described by the histograms derived from the best-fit values using the 600 randomly created data sets. The errors were estimated by a $1 \sigma$-Gauss fit to the histogram of each fit parameter. We fit all near- and mid-infrared visibilities and the SED of UX Ori simultaneously.

\section{Results}

The best-fit geometric $K$-band model consists of an inclined ring and an elongated halo structure, implemented as an elongated Gaussian. We derived a flux fraction of the extended halo of $f_{\mathrm{h}}=16.8 \pm 4.1 \%$ of the total flux. The obtained $K$-band ring fit radius of $0.45 \pm 0.07 \mathrm{AU}$ agrees well with the predicted dust sublimation radius of $\sim 0.44 \mathrm{AU}$ for a dust sublimation temperature of $1500 \mathrm{~K}$ assuming large grains $(\sim 1 \mu \mathrm{m}$; Eq. (1) in Monnier et al. $2005)$, but this does not mean that no smaller grains exist because the dust sublimation radius is characterized by the largest grains that could survive closest to the central star. This assumption of the maximum grain size agrees with grain sizes $(0.75-1.0 \mu \mathrm{m})$ found in former mid-infrared spectral observations of UX Ori (Reimann et al. 1997). The inclination of the ring is $55.6 \pm 2.4^{\circ}$, which corresponds to an elongation axis ratio of $\sim 2$ of the Gaussian halo distribution with an HWHM of $\sim 4.8$ AU. The PA of the system axis (semi-minor axis) is $127.5 \pm 24.5^{\circ}$.

The $N$-band visibilities were modeled with a simple twodimensional Gaussian (see Sect. 3.1.2) with an HWHM of 5.1 \pm $0.1 \mathrm{AU}$ and an elongation ratio of $3.4 \pm 1.0$ (corresponding to an inclination angle of about $72.9 \pm 17.9^{\circ}$ ). This is slightly higher, but consistent within the error bars with the inclination found in the near-infrared of $55.6 \pm 2.4^{\circ}$. The PA of the system axis was fixed to $127.5^{\circ}$ (as found in the near-infrared). We note that due to the sparse $u v$ coverage in the $N$ band, the derived inclination only represents a trend and not a reliable estimate. Further mid-infrared interferometric observations (e.g., with second generation MATISSE instrument) are required to measure the mid-infrared elongation more precisely.

To simultaneously fit the $H$-, $K$-, and $N$-band visibilities and the SED of UX Ori, we applied a modified Chiang \& Goldreich model (DDN, using the cgplus software, Dullemond et al. 2001) that consists of a passive flared dust+gas disk with an inner hole. To account for extended emission, we added an overresolved halo component. In the best-fit model, the puffed-up inner rim has a temperature of $T_{\text {in }}=1498 \pm 70 \mathrm{~K}$. This corresponds, following Eq. (14) in Dullemond et al. (2001), to an inner disk radius of $0.46 \pm 0.06 \mathrm{AU}$ with a rim scale height factor of $\chi=4.02 \pm 0.12$ (defined as the ratio of the disk surface height to the pressure scale height). The disk extends to $R_{\text {out }}=25.9 \pm 5.8$ AU with a surface density exponent of $\beta=-1.79 \pm 0.12$ following the profile $\Sigma(r)=\Sigma_{0}\left(r / r_{\text {ref }}\right)^{\beta}$ (where $r_{\text {ref }}$ defines the radius where $\left.\Sigma\left(r_{\text {ref }}\right)=\Sigma_{0}\right)$. The dust + gas mass of the disk is $M_{\text {disk }}=0.60 \pm 0.04 M_{\odot}$. The extended halo component contributes to the best-fit model with a flux ratio of $f_{\mathrm{h}}=0.13 \pm 0.02$. The inclination and PA of the disk system was found to be $\theta_{\mathrm{DDN}}=70 \pm 5^{\circ}$ and $\Phi_{\mathrm{DDN}}=133 \pm 5^{\circ}$. The error bars represent only the statistical errors. The true total error bars might be a factor of 2-3 larger because of several model specific assumptions (e.g., disk geometry and rim shape) and systematic errors.

\section{Conclusions}

We presented the first interferometric observations and simultaneous modeling of the near- and mid-infrared interferometric measurements and the SED of the prototype star of its class of variable stars, UX Orionis. The $\mathrm{H}$-, $\mathrm{K}$-, and $\mathrm{N}$-band visibilities as well as the SED of UX Ori were modeled with a geometric ring and a modified Chiang-Goldreich disk model with an inner hole (DDN model). The inclination and PA of the system axis of the best-fit DDN model are $70 \pm 5^{\circ}$ and $133 \pm 5^{\circ}$, respectively. The intrinsic $V$-band polarization angle in deep minima might be used as an indicator for the approximate orientation of the symmetry axis of the circumstellar disk (Grinin et al. 1991). Polarimetric measurements in deep minima of UX Ori show a linear $V$-band polarization angle of $125.5-128.7^{\circ}$ (Voshchinnikov et al. 1988), which agrees well with the PA of the system axis that we derived above. The extended halo component contributes with $13 \pm 2 \%$ (DDN model) $/ 16 \pm 4 \%$ (geometric model) to the total flux, similar as found in other young stellar objects with extended halo emission ( 9-16\%, e.g. Monnier et al. 2006; Chen et al. 2012; Vural et al. $2012,2014 a)$ that is interpreted as scattered emission from the outer disk surface or material outside the disk plane, for example, a dusty disk wind (e.g., Bans \& Königl 2012).

The obtained disk inclination angle of $70 \pm 5^{\circ}$ (DDN model) for UX Ori agrees with former modeling studies that used spatially unresolved techniques and found an inclination angle of the UX Ori disk $>=70^{\circ}$ (Voshchinnikov et al. 1988; Natta et al. 1999). With our study on UX Ori, there is now a sample of at least five UXORs whose inclination has been directly measured (VV Ser: $\sim 70^{\circ}$, Pontoppidan et al. 2007; KK Oph: $\sim 70^{\circ}$, Kreplin et al. 2013; CQ Tau: $\sim 30-50^{\circ}$, Chapillon et al. 2008; Eisner et al. 2004; V1026 Sco: 50 , Vural et al. 2014b). In all cases, intermediate- to high-inclination angles were found, which supports the hypothesis that UXOR variability is associated with obscuration events within the disk or disk atmosphere, caused for example by disk material lifted up out of the disk surface that passes through the line of sight. However, the trend of different apparent inclinations in different wavelength bands that emit predominantly in the disk atmosphere indicates that 

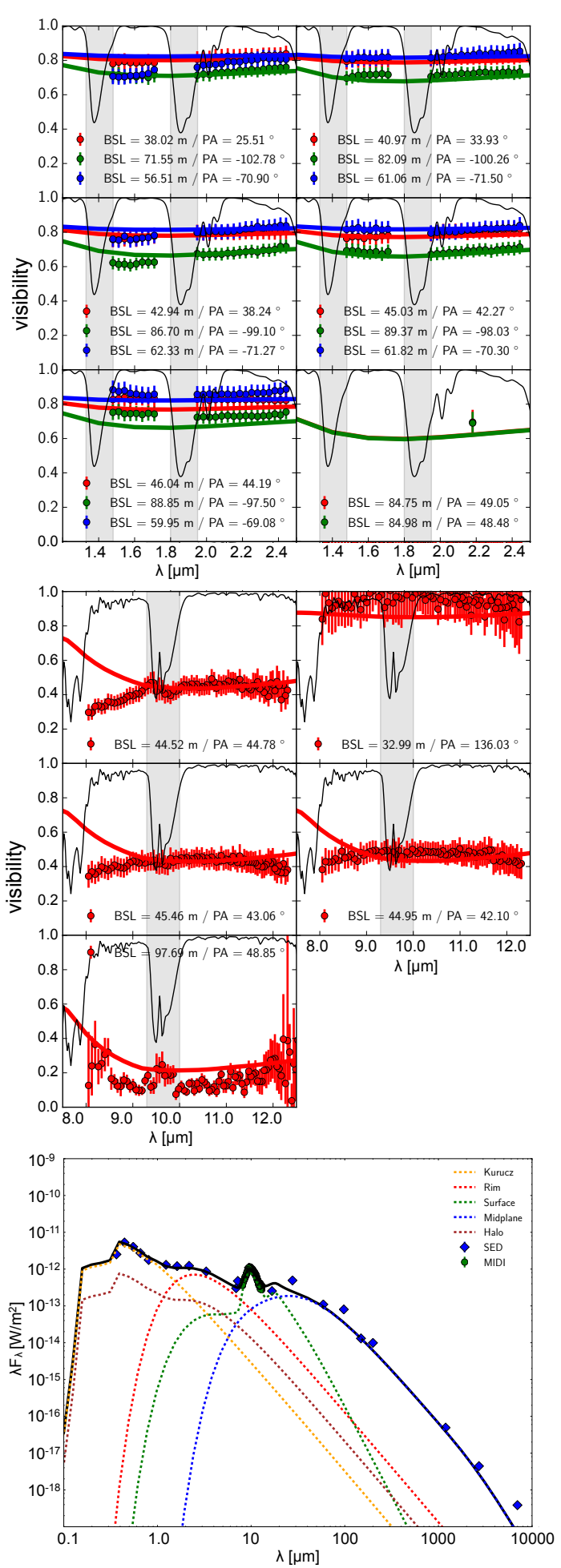

Fig. 4. Simultaneous fit of the visibilities (top) and the SED (bottom) of UX Ori with a CG-disk model including an inner dust-free hole and a puffed-up inner rim. Top: comparison of the best-fit model visibilities (colored lines) to the near- and mid-infrared visibilities (data points) of UX Ori. The shadowed areas denote the telluric band gaps between the $J$ and $H$ and $H$ and $K$ band for the near-infrared (top) and the ozone feature for the mid-infrared (middle) where no data can be taken or where they are not reliable as a result of the limited transmission of the atmosphere (black line). Bottom: spectral energy distribution of UX Ori (data points) with the total flux and the single flux components of the best-fit model. for the most precise measurement of the disk orientation and inclination, observations at longer wavelength (tracing the cooler material in the midplane of the disk) are required. Such observations would be especially important in cases when the infrared interferometry gives the disk inclination with a large uncertainty (e.g., CQ Tau).

Acknowledgements. This research has made use of NASA's Astrophysics Data System Bibliographic Services. We thank the ESO staff at Paranal for their support during the observations. We acknowledge support from an STFC Rutherford Fellowship (ST/J004030/1), Rutherford Grant (ST/K003445/1) and Marie Sklodowska-Curie CIG grant (Ref. 618910). V.G. acknowledge support from the RFBR grant 15-02-09191. L.T. acknowledge support from the RFBR grant 15-02-05399. We are also grateful to Dmitry Shakhovskoi for the photometric support of the interferometric observations.

\section{References}

Bans, A., \& Königl, A. 2012, ApJ, 758, 100

Bertout, C., Robichon, N., \& Arenou, F. 1999, A\&A, 352, 574

Borde, P., Coudé du Foresto, V., Chagnon, G., \& Perrin, G. 2002, VizieR On-line Data Catalog: $\mathrm{J} / \mathrm{A}+\mathrm{A} / 339 / 183$

Chapillon, E., Guilloteau, S., Dutrey, A., \& Piétu, V. 2008, A\&A, 488, 565

Chen, L., Kreplin, A., Wang, Y., et al. 2012, A\&A, 541, A104

Chiang, E. I., \& Goldreich, P. 1997, ApJ, 490, 368

de Wit, W. J., Grinin, V. P., Potravnov, I. S., et al. 2013, A\&A, 553, L1

Dominik, C., Dullemond, C. P., Waters, L. B. F. M., \& Walch, S. 2003, A\&A, 398, 607

Draine, B. T., \& Lee, H. M. 1984, ApJ, 285, 89

Dullemond, C. P., Dominik, C., \& Natta, A. 2001, ApJ, 560, 957

Dullemond, C. P., van den Ancker, M. E., Acke, B., \& van Boekel, R. 2003, ApJ, 594, L47

Eisner, J. A., Lane, B. F., Hillenbrand, L., Akeson, R., \& Sargent, A. I. 2004, ApJ, 613, 1049

Grinin, V. P. 1988, Sov. Astron. Lett., 14, 27

Grinin, V. P. 2000, in Disks, Planetesimals, and Planets, eds. G. Garzón, C. Eiroa, D. de Winter, \& T. J. Mahoney, ASP Conf. Ser., 219, 216

Grinin, V. P., Kiselev, N. N., Chernova, G. P., Minikulov, N. K., \& Voshchinnikov, N. V. 1991, Ap\&SS, 186, 283

Herbst, W., \& Shevchenko, V. S. 1999, ApJ, 118, 1043

Herbst, W., Herbst, D. K., Grossman, E. J., \& Weinstein, D. 1994, AJ, 108, 1906

Kishimoto, M., Hönig, S. F., Antonucci, R., et al. 2011, A\&A, 536, A78

Kreplin, A., Kraus, S., Hofmann, K.-H., et al. 2012, A\&A, 537, A103

Kreplin, A., Weigelt, G., Kraus, S., et al. 2013, A\&A, 551, A21

Kurucz, R. L. 1979, ApJS, 40, 1

Lafrasse, S., Mella, G., Bonneau, D., et al. 2010, VizieR On-line Data Catalog: II $/ 300$

Manoj, P., Bhatt, H. C., Maheswar, G., \& Muneer, S. 2006, ApJ, 653, 657

Mathis, J. S., Rumpl, W., \& Nordsieck, K. H. 1977, ApJ, 217, 425

Menu, J., van Boekel, R., Henning, T., et al. 2015, A\&A, 581, A107

Monnier, J. D., Millan-Gabet, R., Billmeier, R., et al. 2005, ApJ, 624, 832

Monnier, J. D., Berger, J.-P., Millan-Gabet, R., et al. 2006, ApJ, 647, 444

Natta, A., Grinin, V. P., Mannings, V., \& Ungerechts, H. 1997, ApJ, 491, 885

Natta, A., Prusti, T., Neri, R., et al. 1999, A\&A, 350, 541

Petrov, R. G., Malbet, F., Weigelt, G., et al. 2007, A\&A, 464, 1

Pontoppidan, K. M., Dullemond, C. P., Blake, G. A., et al. 2007, ApJ, 656, 980

Reimann, H.-G., Guertler, J., Friedemann, C., \& Kaeufl, H. U. 1997, A\&A, 326, 271

Rostopchina, A. N. 1999, Astron. Rep., 43, 113

Tatulli, E., Millour, F., Chelli, A., et al. 2007, A\&A, 464, 29

Testi, L., Natta, Shepherd, D. S., \& Wilner, D. J. 2001, ApJ, 554, 1087

Vinković, D., \& Jurkić, T. 2007, ApJ, 658, 462

Voshchinnikov, N. V., Grinin, V. P., Kiselev, N. N., \& Minikulov, N. K. 1988, Astrophys., 28, 182

Vural, J., Kreplin, A., Kraus, S., et al. 2012, A\&A, 543, A162

Vural, J., Kraus, S., Kreplin, A., et al. 2014a, A\&A, 569, A25

Vural, J., Kreplin, A., Kishimoto, M., et al. 2014b, A\&A, 564, A118

Wheelwright, H. E., Oudmaijer, R. D., \& Goodwin, S. P. 2010, MNRAS, 401, 1199 